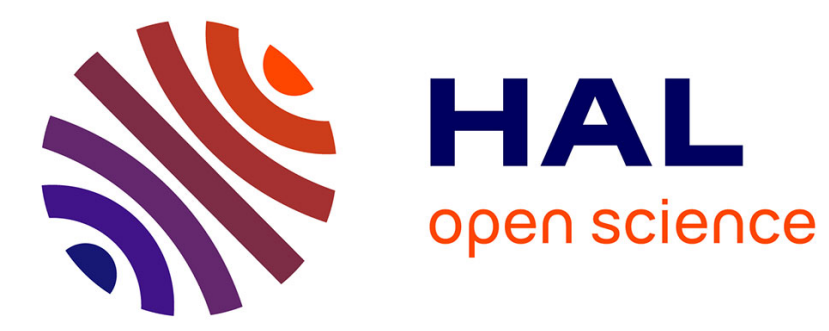

\title{
Shell Gap Reduction In Exotic N = 17 Nuclei
}

A. Obertelli, N. Alamanos, M. Alvarez, F. Auger, R. Dayras, A. Drouart, G. de France, A. Gillibert, B. Jurado, N. Keeley, et al.

\section{To cite this version:}

A. Obertelli, N. Alamanos, M. Alvarez, F. Auger, R. Dayras, et al.. Shell Gap Reduction In Exotic $\mathrm{N}=17$ Nuclei. Frontiers in Nuclear Structure, Astrophysics, and Reactions - FINUSTAR, Sep 2005, Isle of Kos, Greece. pp.177-180, 10.1063/1.2200921 . in2p3-00087146

\section{HAL Id: in2p3-00087146 https://hal.in2p3.fr/in2p3-00087146}

Submitted on 4 Jan 2007

HAL is a multi-disciplinary open access archive for the deposit and dissemination of scientific research documents, whether they are published or not. The documents may come from teaching and research institutions in France or abroad, or from public or private research centers.
L'archive ouverte pluridisciplinaire HAL, est destinée au dépôt et à la diffusion de documents scientifiques de niveau recherche, publiés ou non, émanant des établissements d'enseignement et de recherche français ou étrangers, des laboratoires publics ou privés. 


\title{
Shell Gap Reduction In Exotic $\mathbf{N}=17$ Nuclei
}

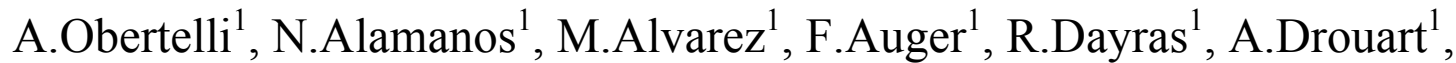 \\ G. de France ${ }^{2}$, A.Gillibert ${ }^{1}$, B.Jurado ${ }^{2}$, N.Keeley ${ }^{1}$, V.Lapoux ${ }^{1}$, W.Mittig ${ }^{2}$, \\ X.Mougeot ${ }^{1}$, L.Nalpas ${ }^{1}$, A.Pakou ${ }^{3}$, N.Patronis ${ }^{3}$, E.Pollacco ${ }^{1}$, F.Rejmund ${ }^{2}$, \\ M.Rejmund ${ }^{2}$, P.Roussel-Chomaz ${ }^{2}$, H.Savajols ${ }^{2}$, F.Skaza $^{1}$, Ch. Theisen ${ }^{1}$ \\ 1- DSM/DAPNIA/SPhN, CE Saclay, F- 91191 Gif sur Yvette, France \\ 2 - Ganil BP 5027, F-14076 Caen Cedex 5, France \\ 3 - Department of physics, The University of Ioannina, 45110 Ioannina, Greece
}

\begin{abstract}
Ne}$ has been investigated through the one neutron transfer reaction ${ }^{26} \mathrm{Ne}(\mathrm{d}, \mathrm{p}){ }^{27} \mathrm{Ne}$ in inverse kinematics at $9.7 \mathrm{MeV} /$ nucleon. The results support the existence of a low lying negative parity state in ${ }^{27} \mathrm{Ne}$ which is a signature of a reduced sd-fp shell gap in the $\mathrm{N}=16$ neutron rich region, at variance with stable nuclei.
\end{abstract}

Keywords: Transfer reaction, inverse kinematics.

PACS: 21.10.Pc, 25.60.Je, 25.70.Hi, 27.30+t, 29.30.Kv;

$\mathrm{N}=16$ has been recently suggested to be magic near the neutron drip line, both theoretically [1] and experimentally [2]. This may arise from an enhancement with isospin of the gap between the $\mathrm{s}_{1 / 2}$ and $\mathrm{d}_{3 / 2}$ subshells of the sd neutron shell. The study of the single particle excitations in $\mathrm{N}=17$ isotones is well suited to determine the spacing between the $\mathrm{d}_{3 / 2}$ subshell and the fp shell. The lowest excitation energies of the negative parity states for the most bound $\mathrm{N}=17$ isotones $\left({ }^{35} \mathrm{Ar},{ }^{33} \mathrm{~S}\right.$ and $\left.{ }^{31} \mathrm{Si}\right)$ are quite high, around $3.5 \mathrm{MeV}$, but below $1.5 \mathrm{MeV}$ for ${ }^{29} \mathrm{Mg}$ [3]. The next $\mathrm{N}=17$ isotone is ${ }^{27} \mathrm{Ne}$ for which very little [4] information are available.

We used the Spiral beam ${ }^{26} \mathrm{Ne}$ at $9.7 \mathrm{MeV} /$ nucleon of the GANIL facility to study the one neutron transfer ${ }^{26} \mathrm{Ne}(\mathrm{d}, \mathrm{p}){ }^{27} \mathrm{Ne}$ in inverse kinematics on a $1 \mathrm{~mm}$ thick solid cryogenic deuterium target [5]. The ejectiles were detected and identified with the VAMOS magnetic spectrometer [6]. Due to the large momentum acceptance and the inverse kinematics, not only ${ }^{27} \mathrm{Ne}$ was transmitted, but others ejectiles simultaneously observed in the focal plane from others reaction channels like $(d, d ')$ or $(d, t)$. A complete identification was obtained with usual combinations of time of flight, energy loss and transverse position in the focal plane (fig.1). Due to the target thickness, the recoiling protons emitted at backward angles in the lab frame were not measured.

In coincidence, the emitted photons were measured with part of the EXOGAM spectrometer in a compact geometry around the target. We used 11 clovers, placed at distances varying from 11 to $17 \mathrm{~cm}$ from the center of the target in three different rings depending on the polar angles $\theta=45,90$ and 135 degrees in the laboratory frame. The intrinsic resolution of the whole system was measured to be $2.6 \mathrm{keV}$ (FWHM) at $1332 \mathrm{keV}$, and the photopeak efficiency was estimated to be $4.8 \%$. 


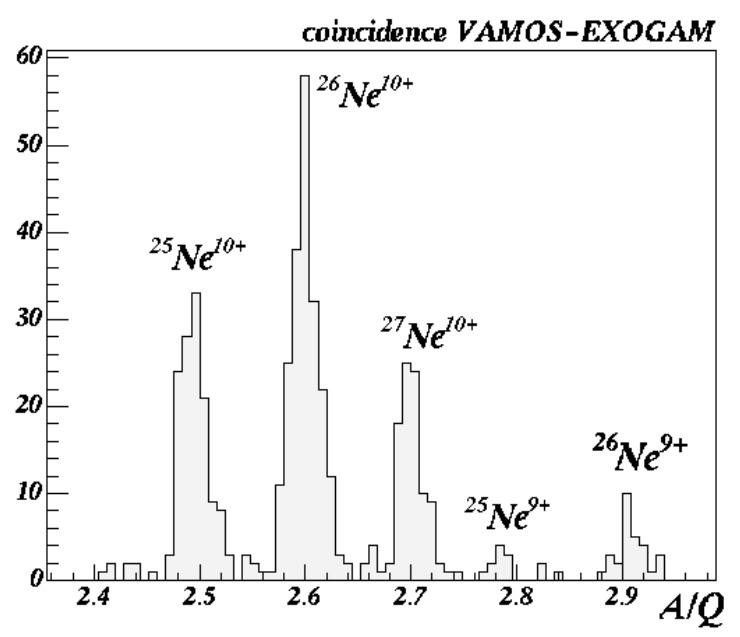

FIGURE 1. Final identification of the ejectiles in the focal plane of the VAMOS spectrometer in coincidence with the photons emitted in the EXOGAM spectrometer.

As the reaction vertex in the target is not reconstructed, only a mean value of the relative velocity $\beta=v / c$ may be used. For ${ }^{27} \mathrm{Ne}$ and the $(d, p)$ channel, a mean value $\beta=$ 0.105 was adopted, for which the measured energies are equally well corrected for the Ge detectors at backward and forward angles.
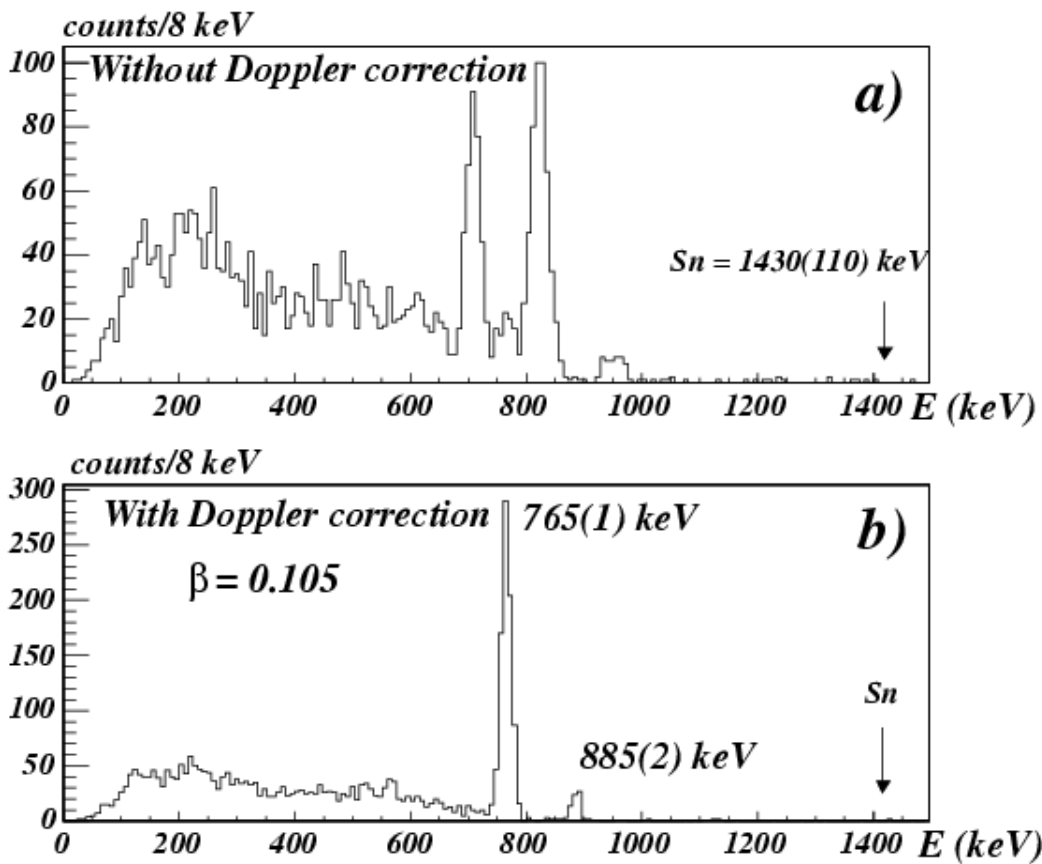

FIGURE 2. Energy spectrum of photons measured in coincidence with ${ }^{27} \mathrm{Ne}$ in the VAMOS spectrometer (a) without and (b) with Doppler correction resulting in two transitions at 765 and 885 $\mathrm{KeV}$. The Doppler shift effect is especially visible for the intense $765 \mathrm{keV}$ transition on (a) with 3 peaks corresponding to the 3 rings at 45, 90 (with a much lower efficiency due to the target) and 135 degrees.

Below the neutron emission threshold $\mathrm{S}_{1 \mathrm{n}}=1430$ (110) $\mathrm{keV}$, two transitions are observed at 765 and $885 \mathrm{keV}$ on the Doppler-corrected spectrum (fig.2b). Within the 
limited statistics, we did not observe any coincidence between these transitions. Due to the low $\mathrm{S}_{1 \mathrm{n}}$ value, it is the most likely that these transitions directly connect two excited states to the ground state as shown on fig.3. We did not observe a strong evidence for a transition at $120 \mathrm{keV}$ over the background, connecting the $885 \mathrm{keV}$ state to the $765 \mathrm{keV}$ state. An upper limit of $20 \%$ was deduced for the branching ratio $\tau$ of such a transition, compared to the direct decay to the ground state. Finally, that transition was observed in a recent knock-out experiment done at MSU with a ${ }^{28} \mathrm{Ne}$ beam, which validates the proposed scheme on fig.3 (left part). On the right part are shown the predictions of the USD shell model [7] for the positive parity states of ${ }^{27} \mathrm{Ne}$. Only one positive parity state is predicted below the neutron threshold, with spin $1 / 2^{+}$. The two experimental levels are consistent with that state, so that one of them might be a negative parity state.

\begin{tabular}{lcc}
\hline $\mathbf{E}(\mathbf{k e V})$ & $\boldsymbol{\sigma}(\mathbf{m b}) \boldsymbol{\tau}=\mathbf{0 \%}$ & $\boldsymbol{\sigma}(\mathbf{m b}) \boldsymbol{\tau}=\mathbf{2 0 \%}$ \\
\hline 0 gs & $4.6(3.9)$ & $4.6(3.9)$ \\
765 & $21.9(2.3)$ & $21.3(2.3)$ \\
885 & $2.5(0.5)$ & $3.1(0.6)$
\end{tabular}

Table 1 : Experimental integrated cross sections for two different values of the branching ratio $\tau, 0$ and $20 \%$, for the decay of the $885 \mathrm{keV}$ state as shown on fig. 3

To go further, we performed calculations with the code FRESCO using the CDCC (Continuum Discretized Coupled Channels), CRC(Coupled Reaction Channels) and CC (Coupled Channels) formalism [8], taking into account the deuteron break-up. The results are in good agreement with the experimental values seen in table 1 with a large spectroscopic factor for the $765 \mathrm{keV}$ state, whatever the transferred angular momentum may be $(\Delta \ell=1$ or 3$)$. Due to the large uncertainties, no direct assignment was done for $\Delta \ell$.

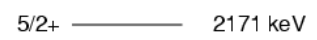

$\mathrm{Sn}=1430(110) \mathrm{keV}$

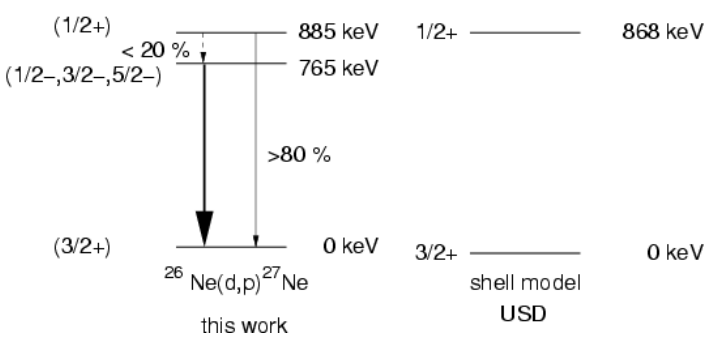

FIGURE 3. Spectroscopy of ${ }^{27} \mathrm{Ne}$ bound states (left part) observed in the one neutron transfer ${ }^{26} \mathrm{Ne}(\mathrm{d}, \mathrm{p})^{27} \mathrm{Ne}$ compared to the USD shell model predictions (right part). 
Under the assumption consistent with the USD shell model that the ground state is $\mathrm{J}^{\pi}=3 / 2^{+}$, our data are consistent with a $1 / 2^{+}$state at $885 \mathrm{keV}$. A negative parity state at $765 \mathrm{keV}$ may be $1 / 2^{-}, 3 / 2^{-}, 5 / 2^{-}$or $7 / 2^{-}$. However, a $7 / 2^{-}$state would decay to the ground state via a M2 transition, resulting in a strong anisotropy forward/backward in the $\gamma$-ray emission not seen in the data, which excludes the $7 / 2^{-}$spin. A $5 / 2^{-}$parity is not excluded by our data, however it would strongly favor a direct decay (M1+E2) of the $885 \mathrm{keV}$ state $1 / 2^{+}$to the ground state compared to a slow $120 \mathrm{keV} \mathrm{M} 2$ transition to the $5 / 2^{-}$state. A summary of our analysis is shown on fig.3.

The low-lying fp shell orbits, suggested for ${ }^{29} \mathrm{Mg}$ and observed in this work for ${ }^{27} \mathrm{Ne}$, can have two origins : a smaller spherical energy gap between the $\mathrm{d}_{3 / 2}$ subshell and the fp shell and (or) deformation.

\section{REFERENCES}

1. T. Otsuka et al., Phys. Rev. Lett. 87, 082502 (2001); A. Obertelli et al., Phys. Rev. C 71024304 (2005),

2. A. Ozawa et al., Phys. Rev. Lett. 84, 5493 (2000),

3. L.K. Fifield et al., Nucl. Phys. A 437, 141 (1985); P. Baumann et al., Phys. Rev. C39, 626 (1989),

4. M. Belleguic-Pigeard de Gurbert, Ph.D Thesis, Université Lyon I, 2000; H. Iwasaki et al., Phys. Lett. B 620, $118(2005)$

5. A. Obertelli et al., to be published in Phys. Lett.B,

6. H. Savajols \& the VAMOS collaboration, Nucl. Phys. A 654, 1027c (1999),

7. B.A. Brown and B.H. Wildenthal, Annu. Rev. Nucl. Part. Sci. 38, 29 (1988),

8. N. Austern et al., Phys. Reports 154, 124 (1987). 